\title{
Aspectos prácticos de implementación en compensadores PID para control de posición de un automóvil

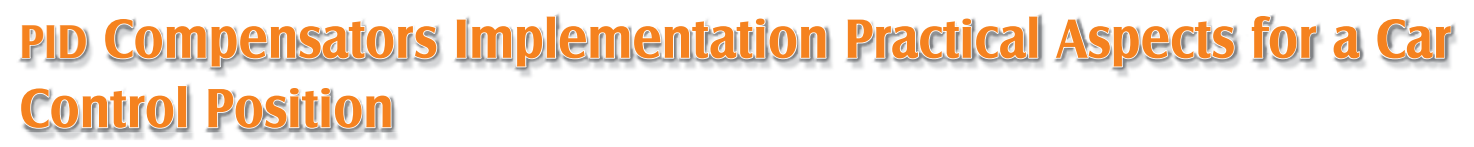

\section{Resumen}

El siguiente artículo tiene la intención de mostrar una herramienta de software con el enfoque de algunos problemas en la implementación de controladores PID. Esta herramienta ha sido desarrollada en el entorno Matlab y permite el análisis de los elementos que se deben tener en cuenta en la ley de control Pid que se implementa en la práctica. También se divide en un conjunto de programas: el primero es una aplicación que se ejecuta en Matlab y el segundo es un bloque de Simulink que se ejecuta en el entorno de Simulink. El primero tiene las herramientas de análisis que permiten la comparación de las estructuras de PID con características prácticas. También es posible importar modelos de diferentes representaciones, tales como: funciones de transferencia y espacio de estado, y más aún un modelo dinámico conjunto de Simulink. Por otra parte, las estructuras clásicas PID discretos o continuos están disponibles, y no es una opción para implementar algoritmos personalizados. Por último, una opción para exportar el controlador a $\mathrm{p}$ / área de trabajo

M. Sc. Ingeniería Electrónica y de Computadores, Universidad de los Andes, M. Sc. Administración, Universidad de los Andes, investigador y profesor, Universidad Distrital FJDC. E-mail: aescobard@udistrital.edu.co

** M. Sc. Ingeniería Electrónica y de Computadores, Universidad de los Andes, M. Sc. Administración, Universidad de los Andes, investigador y profesor, Grupo ICT Research, Universidad de San Buenaventura, Bogotá, E-mail: Ibarrero.edu.co 
como modelo LTI está disponible. Este último es un bloque desarrollado exclusivamente para Simulink / Matlab con las siguientes características: permite al usuario elegir las estrategias para modificar la estructura PID, la configuración del bloque durante la simulación es posible, diferentes estructuras PID se pueden elegir, y hay una opción para implementar algoritmos personalizados. Cualesquiera de los programas donde se desarrollaron abiertamente, es decir, si el usuario desea modificar la interfaz o cambiar el algoritmo, él o ella podría ser capaz de hacerlo. El objetivo principal de lograr es demostrar cómo la ley de control mediante un PID se puede mejorar si se tienen en cuenta las características de aplicaciones prácticas en PID. Por lo tanto, se mejora el sistema general de control a realizar.

\section{Palabras clave}

Implementación, pid, análisis, estrategias, herramientas.

\section{Abstract}

This paper shows a software tool with the approach of some issues in the implementation of PID controllers. This tool has been developed in the MATLAB environment and allows analyzing the elements that have to take into account when the PID control law is implemented in the practice. It is also divided in a set of programs: the first one is an application that works on MATLAB and the second is a Simulink block that works on the Simulink environment. The MATLAB Application has tools of analysis which allow comparing the PID structures with practical features. It is also possible for this application to import models of different representations such as: transfer functions, state space, and even more a whole dynamic model from SIMULINK. Moreover, discrete or continuous classical PID structures are available; and there is an option to implement custom algorithms. FinaIly, an option to export the controller to Matlab/workspace as LTI model is available. The Simulink block was exclusively developed for Simulink/MATLAB with the following features: it allows to the user to choose strategies to modify the PID structure, configuring the block during the simulation. Also different PID structures can be chosen, and there is an option to implement custom algorithms. Either programs were developed as open source code, which means that, if the user wanted to modify the interface or changed the algorithm, he or she might be able to do so. The main goal is to prove how the PID law can be improved when the practical implementation features are taken into account in the practice of PID control. Hence, the overall control system perform is improved.

\section{Keywords}

Implementation features, PID, analysis, strategies, tools.

\section{Introducción}

En la implementación práctica de compensadores PID a nivel industrial, se tienen aspectos de implementación que en la academia no se abarcan con mucha profundidad. En este artículo se presenta un paquete de software desarrollado, el cual permite analizar estas características mostrando las dificultades que se presentan con la ley básica del 
control PID y como se pueden usar estrategias que han sido ya utilizadas para mitigar estos problemas.

El artículo comienza abordando los conceptos básicos de los términos de la ecuación PID; se describen los problemas asociados con cada uno, y se plantean estrategias de solución que permiten mejorar el desempeño del controlador en la práctica. En la siguiente sección de analizará un sistema dinámico para controlar la posición de un automóvil. Este tiene como objetivo comparar un controlador PID básico contra uno práctico y así evaluar sus modos de operación mediante los índices de desempeños que se presentan por cada prueba. Por último, se presentan conclusiones del trabajo realizado.

\section{Conceptos básicos}

A continuación se presentan dos de las formas clásicas de la ley de control del PID [1]:

Estructura ideal

$$
C_{i}=k_{p}\left(1+\frac{1}{T_{i} s}+T_{d} s\right)
$$

Estructura paralela

$$
C_{p}=k_{p}+\frac{k_{i}}{s}+k_{d} s
$$

Debido a algunos problemas asociados en el término tanto integral como derivativo de la ecuación, en la práctica, estas estructuras son modificadas para implementarlas apropiadamente.

$\mathrm{Si}$, por ejemplo, se resuelve (1) se obtiene lo siguiente:

$$
\begin{aligned}
C_{i} & =k_{p}\left(\frac{s+1+T_{i} T_{d} s^{2}}{T_{i} s}\right) \\
C_{i} & =\frac{k_{p} T_{i} T_{d} s^{2}+k_{p} s+k_{p}}{T_{i} s}
\end{aligned}
$$

Naturalmente, se observa que la función de transferencia en (3) es impropia debido a la acción derivativa luego presenta un problema a la hora de realizar su implementación.

En las siguientes secciones se enumeran los problemas más relevantes que se presentan en la implementación de compensadores PID.

\section{Problemas con la acción derivativa}

La acción derivativa tiene la siguiente forma:

$$
D=T d \cdot \frac{d}{d t} e(t)
$$


Al considerar la siguiente función que puede ser una señal de ruido, debido posiblemente, a un elemento sensórico en el lazo de control:

$$
\begin{aligned}
& f(t)=A \operatorname{sen}(\omega \cdot t) \\
& f(t)^{\prime}=A \omega \cos (\omega \cdot t)
\end{aligned}
$$

Si se considera un control de tipo D para esa función, se obtiene lo siguiente

$$
u(t)=A \cdot k_{p} \omega \cos (\omega \cdot t)
$$

Efectivamente, al usar la acción derivativa se obtiene una señal no deseada ocasionando inestabilidad en la señal de control. En la práctica, esta señal podría llegar a causar daño al actuador del sistema explicando porqué en la mayoría de las implementaciones PID, la parte derivativa es desconectada.

\section{Saturación en el modo integrador}

El integrador tiene la siguiente forma:

$$
I=\frac{1}{T i} \cdot \int e(t) \cdot d t
$$

Cuando hay un cambio en la referencia, la parte integral comienza a actuar mientras la señal de error continúe. Si el error es muy grande, el término integral, matemáticamente, se puede hacer muy grande [2]. Esto causa que la señal de control (u), cause que el elemento final de control se sature. Puesto que el actuador físicamente tiene un valor máximo y mínimo (ejemplo, una válvula), estos valores no son suficientes para contrarrestar la señal de error, por lo tanto, la parte integrativa del algoritmo, sigue creciendo o decreciendo hasta eliminar el error. Sin embargo, en el caso particular que una válvula haya llegado a su $100 \%$ de abertura, la habilidad de control se pierde puesto que la válvula no abrirá más de sus limitantes físicas.

Cuando la señal de control no tiene un efecto físico sobre el elemento actuador, es decir, cuando el compensador ha perdido la habilidad para regular el proceso, se dice que el compensador ha entrado en saturación. Como está asociado con el término integral se conoce como saturación en el modo integrativo.

\section{Ponderación en la referencia}

La constante proporcional actúa directamente sobre el error. Esto es:

$$
P=k_{p}(r(t)-y(t))
$$

En una estructura con retroalimentación se desea obtener la mejor respuesta contra las perturbaciones y, al mismo tiempo, una rápida reacción a los cambios de referencia. Esto se puede obtener al aumentar la ganancia Kp del compensador, lo cual, en muchas ocasiones, produce una oscilación en la respuesta paso. 
En la siguiente sección se hablará de las estrategias más comunes usadas para resolver los problemas anteriormente mencionados. Cabe resaltar, que esto trae ventajas como desventajas. Sin embargo, en la práctica, estas soluciones son adoptadas y normalmente se ofrecen estructuras adicionales.

\section{Estrategias para mejorar el desempeño del compensador PID}

Como se mostró en la sección anterior, evidentemente existen unos problemas asociados con ley de control de PID en la práctica. A continuación, se mostrarán las estrategias más adoptadas para superar estas dificultades mediante simulación.

Filtro en la parte derivativa y concepto de la "patada derivativa (kick derivative)»

El uso del término derivativo aumenta el ancho de banda en frecuencia de la señal de control, esto es, permite conocer el próximo estado del error o el proceso (como se verá más adelante). También puede aumentar la estabilidad y mejorar la ganancia en el término integrativo.

Filtro lineal pasa bajo

Como se observó en (6), especialmente si el ambiente es ruidoso, señales de alta frecuencia y alta amplitud, pueden aparecer en la señal de control. La solución más común ofrecida por los fabricantes es utilizar un filtro pasa-bajos de por lo menos de primer orden (N) [3]. La constante de tiempo del filtro es elegida de tal manera que se elimine el ruido y que no tenga un efecto muy significativo sobre la dinámica del compensador. De esta forma, el término derivativo sería

$$
u(t)=k p \frac{T d s}{1+\frac{T d}{N} s}
$$

Teniendo en cuenta esto, tomaremos como modelo la estructura Ideal (ecuación (1)) la cual tendría la siguiente forma [1]:

$$
C_{i}=k_{p}\left(1+\frac{1}{s T_{i}}+\frac{T_{d} s}{\frac{T_{d}}{N} s+1}\right)
$$

El orden del filtro, $\mathrm{N}$, en los PID comerciales, se encuentra en el rango de 1 y 33. Alternativamente, si se necesita una mayor atenuación en altas frecuencias un filtro de segundo orden puede ser adoptado [1]:

$$
k_{p}\left(1+\frac{1}{s T_{i}}+\frac{T_{d} s}{\frac{T_{d}}{N} s+0,5\left(\frac{T_{d}}{N} s\right)^{2}}\right)
$$


Otro problema asociado con la parte derivativa es la "patada derivativa» la cual aparece en los cambios de referencia. Esto produce que el término derivativo tome un valor pico muy alto lo cual es indeseable en la señal de control.

La manera de evitar esto es aplicando la acción derivativa a la variable del proceso y no al error. Esto se puede hacer porque cuando la referencia es estable o constante, la señal de error y proceso son equivalentes pero diferentes en signo.

Consideremos lo siguiente [2]:

$$
\begin{aligned}
& e=r-y \\
& \frac{d}{d t} e(t)=\frac{d}{d t}(r-y) \\
& \frac{d}{d t} e(t)=-\frac{d}{d t} y(t)
\end{aligned}
$$

Donde e, es el error; $y$, es la variable del proceso (process variable y $r$, es la referencia (set point)

Para el término derivativo entonces se tiene:

$$
u(t)=-k d \frac{d}{d t} y(t)
$$

Debido a que la derivada del error y la del proceso son equivalentes en todos los instantes de tiempo excepto en los cambios de referencia, esto no afecta las constantes kp, Ti y Td del compensador. También, en ambos casos, el rechazo a las perturbaciones en el sistema es el mismo.

Con esta modificación, el diagrama de bloques quedaría así:

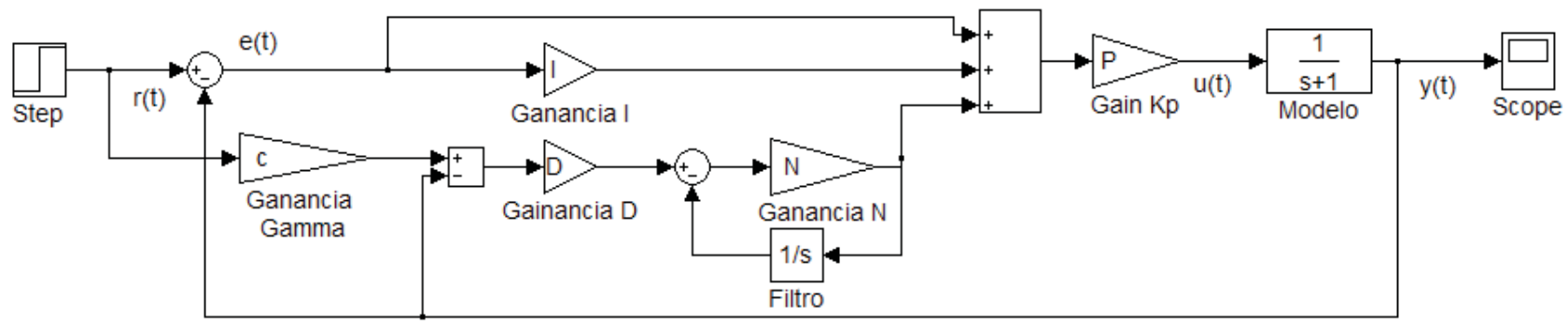

Figura 1. Control PID con filtro en la derivada actuando sobre la señal de proceso.

La señal de error es e $(t)$, la entrada es $r(t)$, la señal de control es $u(t)$ y el proceso es y $(t)$. Se observa el parámetro $D$ (derivada) en cascada con un filtro de primer orden. El parámetro $C$, en este caso, permite seleccionar si la derivada se aplica directamente al error o al proceso; y $\mathrm{N}$ permite ajustar la frecuencia de corte. Ambas opciones están disponibles en la aplicación.

Estrategias para minimizar el impacto de la saturación en el modo integrador [1] [10] 


\section{Evitar la saturación}

Aunque es una manera intuitiva de realizarlo, es posible evitar que el término integrativo se acumule limitando la señal de control. Sin embargo, esto requiere que de alguna manera, el diseño del compensador tenga un esfuerzo adicional y, lo más importante, es que si el compensador no llega propiamente a autorregularse, puede traducirse en un muy bajo desempeño. En un caso práctico esto es indeseable.

\section{Condicionar el integrador}

Una clásica manera de realizarlo es condicionando el integrador. Esto es, apagar el integrador cuando haya alcanzado una cierta condición. Basado en esto, se puede tener lo siguiente:

- Limitar el valor del integrador;

- Apagar el integrador cuando la señal de error alcance un valor de condición, esto es, cuando el valor de la señal del proceso se encuentre lejos de la referencia;

- Se detiene la integración cuando $u \neq u^{\prime}$;

- Se detiene la integración cuando la señal de control se satura y la señal de proceso y el error tienen el mismo signo. Esto es: $u \cdot e>0$

Los primeros dos métodos presentan una desventaja. En el primer método se deben elegir los valores de tal manera que permitan llegar a la referencia. De lo contrario, se podría tener un error de estado estacionario. En el segundo caso, se debe prevenir que el compensador se estanque ya que la señal de error puede ser muy grande con respecto al valor condicional. Además, incluyen un diseño adicional no siendo muy deseable para la sencillez que permite el PID.

El tercer y cuarto método evitan estos problemas. El último método es una extensión mejorada del tercer método lo que la convierte en una solución más viable.

El diagrama de bloques sería el siguiente:

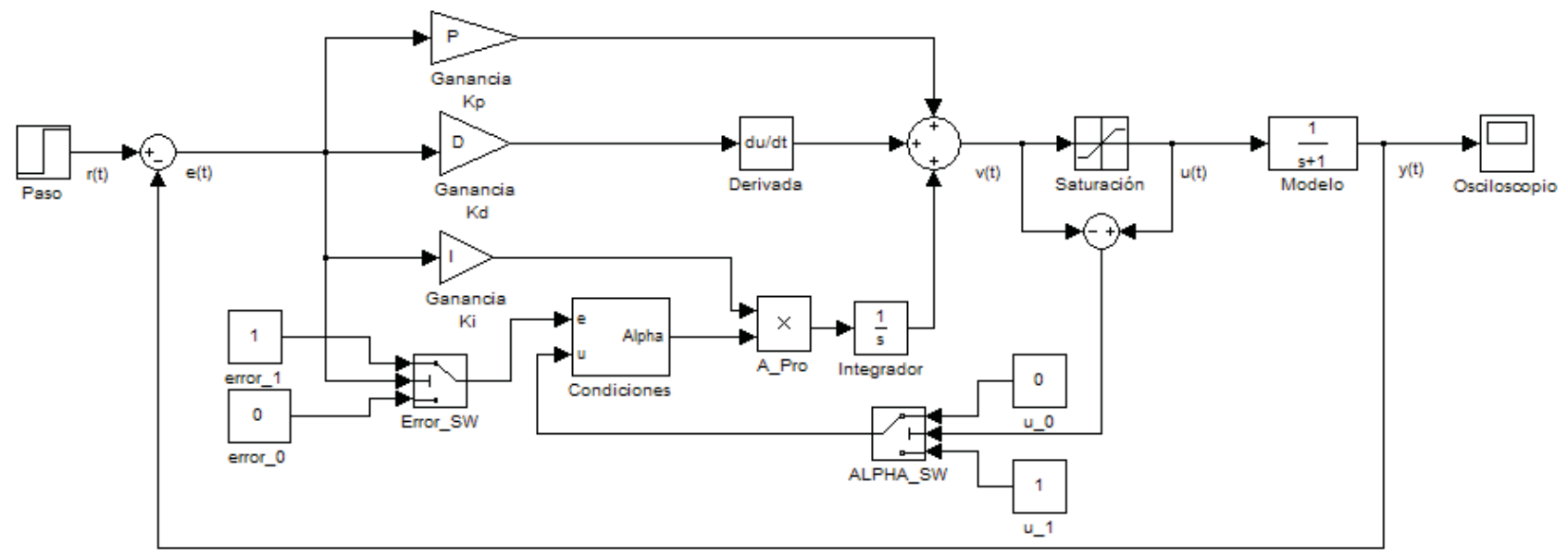

Figura 2. Estrategia para evitar la saturación en el término integral. 
La figura 2 muestra un sistema de control PID, con el término I modificado. En el bloque Condiciones se determina la salida, se debe activar la integral o no dependiendo de los valores del error y de la señal de control.

El algoritmo que describe el método Factor alpha (de aquí en adelante ese será el nombre) se encuentra a continuación:

$$
e_{i}=\left\{\begin{array}{llllll}
k i \cdot e \cdot \alpha & \alpha=0, & \text { si } & u>u \max & y & e>0 \\
k i \cdot e \cdot \alpha & \alpha=1, & \text { si } & u>u \max & y & e<0 \\
k i \cdot e \cdot \alpha & \alpha=1, & \text { si } & u<u \min & y & e>0 \\
k i \cdot e \cdot \alpha & \alpha=0, & \text { si } & u<u \min & y & e<0 \\
k i \cdot e \cdot \alpha & \alpha=1, & \text { otro }- \text { caso } & & &
\end{array}\right.
$$

\section{Respaldo para el valor integrativo (Back-calculation)}

Esta técnica trata de recalcular el término integrativo (Back Calculation por su término en inglés) cuando el actuador se satura. Esto es:

$$
e_{i}=\frac{K p}{T i}+\frac{1}{T_{t}} \cdot\left(u^{\prime}-u\right)
$$

Donde $u$ ' es la salida del compensador; $u$ es la salida parcial del compensador antes del saturador; Tt es una constante llamada constante de seguimiento de tiempo (Tracking time constant).

El valor de Tt es el encargado de ajustar el valor del término integrativo así que su elección es importante para el desempeño del controlador.

Se han propuesto diferentes métodos de ajuste que permiten sintonizar este valor. Esto es,

$$
T_{t}=\sqrt{T_{d} \cdot T_{i}}
$$

Sin embargo, cuando la estructura es de tipo PID esta solución no se puede adoptar. En ese caso, entonces

$$
T_{t}=T_{i}
$$

El diagrama de bloques con esta técnica sería el siguiente:

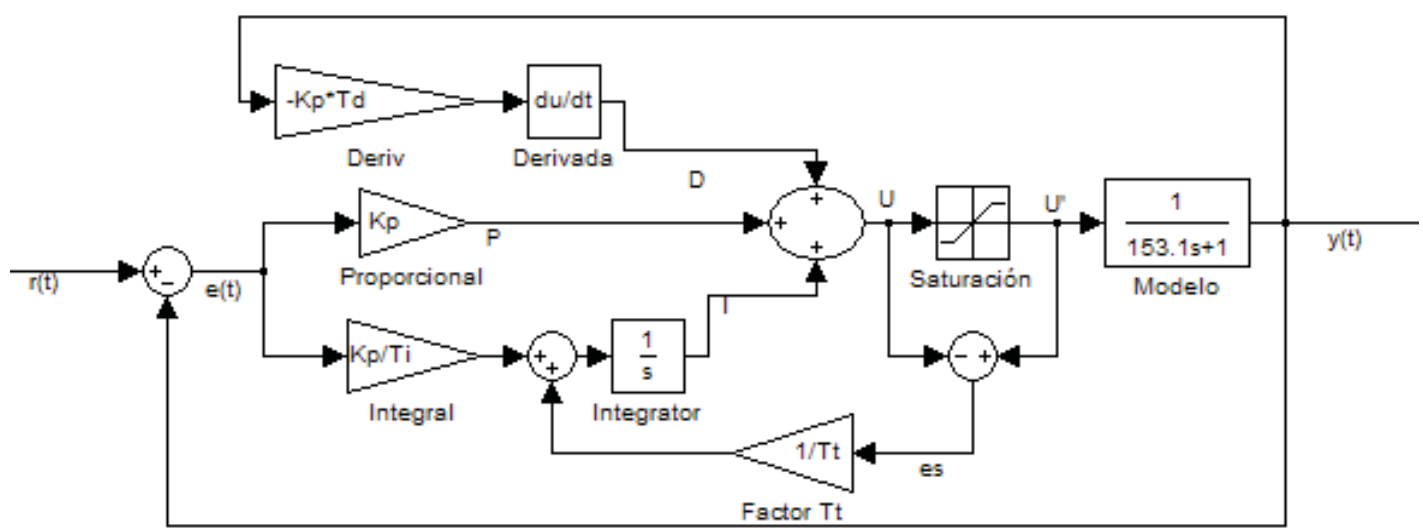

Figura 3. Estrategia para evitar la saturación en el término integral. La figura muestra un sistema de control PID para la planta en el bloque modelo. 


\section{Ponderación referencia}

Si se trata de obtener un alto rechazo a la carga debido a las perturbaciones del sistema, una solución bastante viable es ponderar la referencia mediante un factor llamado beta, así:

$$
P=k_{p}(\beta \cdot r(t)-y(t))
$$

Como el factor beta actúa directamente sobre la referencia, es posible diseñar un sistema de control con 2 grados de libertad como se muestra en el siquiente diagrama de bloques:

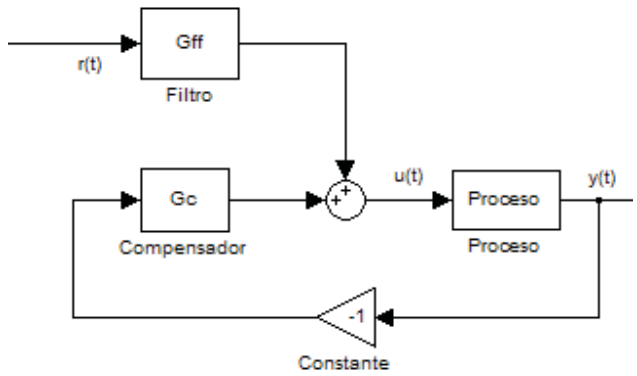

Figura 4. Sistema de control PID con 2 grados de libertad

Se observa que la función de transferencia desde el setpoint $r(t)$ hasta el controlador u(t) está dada por la forma:

$$
G_{f f}(s)=k p \cdot\left(\beta+\frac{1}{s T_{i}}+s T_{d}\right)
$$

Y la función de transferencia desde la variable de proceso y(t) hasta la señal de control $\mathrm{u}(\mathrm{t})$ es:

$$
G_{c}(s)=k p \cdot\left(1+\frac{1}{s T_{i}}+s T_{d}\right)
$$

Como en este caso el factor beta afecta a la referencia entonces se conoce como ponderación de la referencia.

Otra estructura alternativa a la anterior es la mostrada en la figura 5 en este caso con un controlador en paralelo (2).

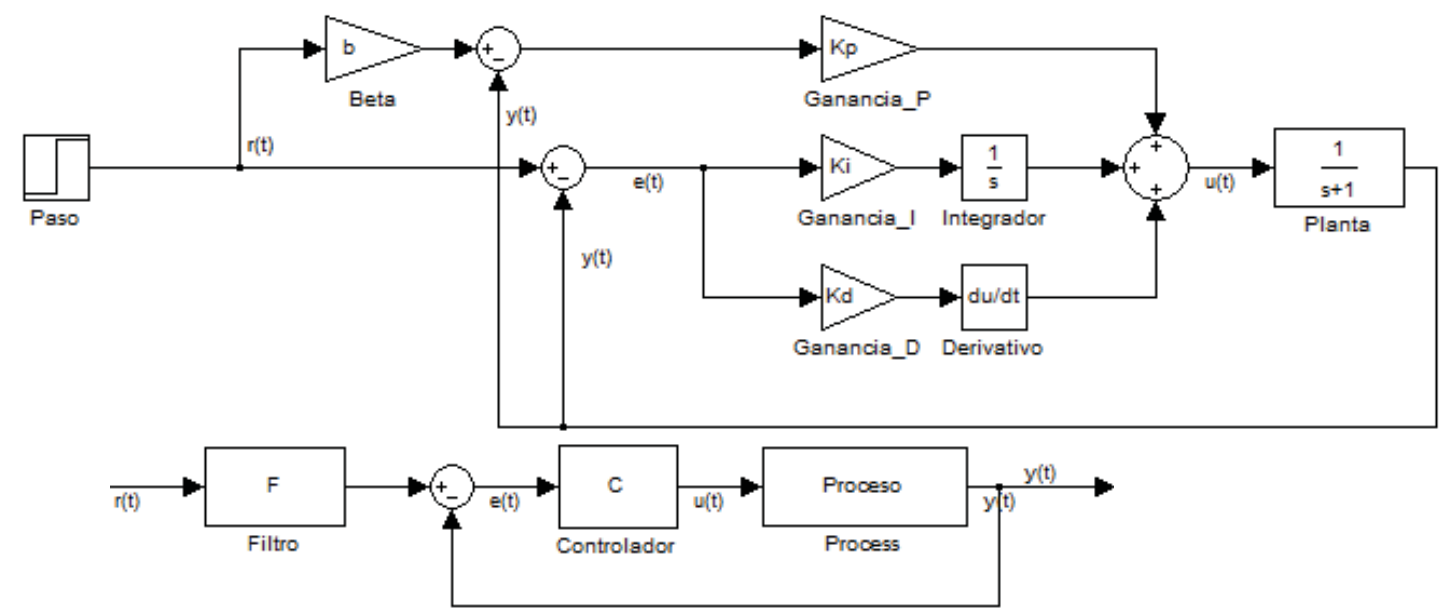

Figura 5. Diagrama de bloques con ponderación en la referencia. 
En la figura (a) se muestra un controlador PID en estructura paralela; $r(t)$ es la señal de entrada; $y(t)$ el proceso; e(t) el error; $u(t)$ la señal de control. En (b) se muestra una estructura alternativa; $F$ es el pre-filtro a la entrada de la referencia; $C$ es el controlador PID; Proceso es el modelo de la planta.

$$
C(s)=k_{p}+\frac{k_{i}}{s}+k_{d} s
$$

y

$$
F(s)=\frac{\left(\beta \cdot k_{p}+k_{d}\right) \cdot s^{2}+\left(\beta \cdot k_{p}+k_{i}\right) \cdot s+k_{i}}{\left(k_{p}+k_{d}\right) s^{2}+\left(k_{p}+k_{i}\right) \cdot s+k_{i}}
$$

donde $0<\beta<1$

Los diagramas anteriormente mostrados se conocen como: configuración de la forma feedforward/feedback [1] con un factor de ponderación llamado beta.

\section{Estructura práctica de controlador PID}

Si todas las modificaciones anteriores se tienen en cuenta, la ecuación del controlador PID quedaría expresada de la siguiente manera:

$$
\begin{gathered}
u(t)=K_{p}\left(\beta \cdot r(t)-y(t)+\frac{1}{T_{i}} \int_{0}^{t} e(t) d t+T_{d}\left(d\left(\gamma \cdot r(t)-y_{f}(t)\right)\right)\right) \\
\frac{T_{d}}{N} \frac{d y_{f}(t)}{d t}=y(t)-y_{f}(t)
\end{gathered}
$$

Esta estructura es comúnmente conocida como: controlador pid de la forma ISA [1]. En general, el Beta toma valores entre 0 y 1 , indicando si se aplica o no la parte proporcional a la referencia, y el Gamma, que varía también entre 0 y 1 , indica si se deriva la señal de proceso o la de error. Esta expresión se conoce también como controlador beta-gamma.

El diagrama de bloques es el que se presenta en la figura 6.

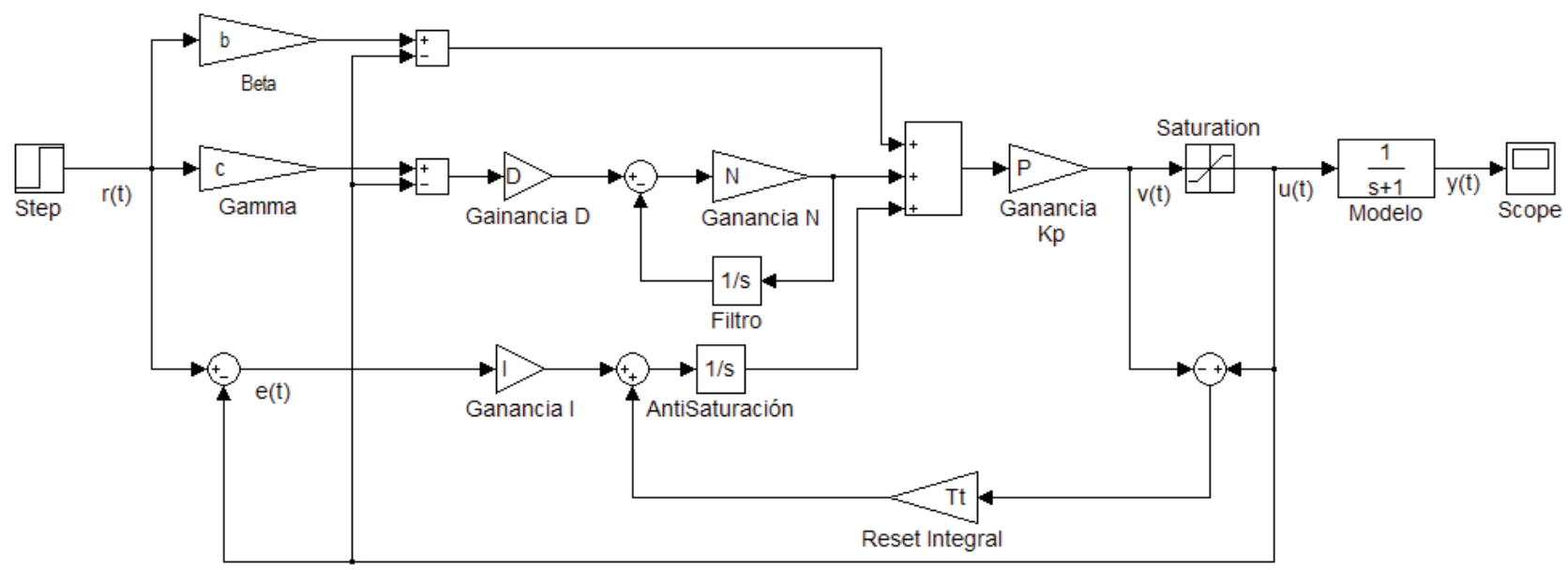

Figura 6. Implementación práctica del PID. 
Cada término se encuentra con las modificaciones correspondientes: los bloques Beta y Gamma corresponden respectivamente la parte proporcional y derivativa; el filtro derivativo se encuentra en el bloque ganancia $N$ junto con el bloque Filtro; el término integral ha sido modificado y ahora se encuentra con el algoritmo de antisaturación.

\section{Experimentos y resultados}

Se considera un sistema de control de velocidad en un automóvil cuyas ecuaciones dinámicas son las siguientes:

$$
\begin{gathered}
F_{d}=M \frac{d v}{d t}+F a+F g \\
F g=M_{g} \operatorname{sen} \theta \\
F a=C_{a}\left(v+v_{w}\right)^{2}
\end{gathered}
$$

Donde $M(d v / d t)$ es la fuerza de inercia, $F_{a}$ es la resistencia aerodinámica y $F_{g}$ es la resistencia de ascensión o fuerza descendente. Las fuerzas $F_{d}, F_{a}$ y $F_{g}$ se producen como se muestra en las ecuaciones en (24), donde $v_{w}$ es la velocidad de las ráfagas de viento, $M$ es la masa del vehículo y pasajero(s), $\theta$ es el ángulo de inclinación de la carretera y $\mathrm{C}_{a}$ es el coeficiente de resistencia aerodinámica.

El diagrama de bloques utilizado para realizar los experimentos es el que se encuentra a continuación:

Se proponen 2 experimentos con las siguientes configuraciones para el bloque y con ello: obtener las respuestas e índices de desempeño para comparar los resultados.

En el experimento 1: Figuras 8(a) - 10(a); los parámetros de configuración del bloque son: PID estructura ideal, tiempo de muestreo $0.01 \mathrm{~s} ; \mathrm{Kp}=0.6 \mathrm{Ti}=12 \mathrm{Td}=0.6$ Beta $=0$, $\mathrm{N}=5$.

En el experimento 2: Figura 8 (b) - 10 (b); los parámetros de configuración del bloque son: PID estructura ideal, tiempo de muestreo 0,01s; $\mathrm{Kp}=0.6 \mathrm{Ti}=12 \mathrm{Td}=0.6$.

Todas las constantes fueron halladas por el método de Ziegler- Nichols.

En las pruebas que se realizaron y en la evaluación de desempeño para ambos controladores, varios ítems revelan que el PID con las características de implementación (práctico) muestra un mejor desempeño que el PID básico. Por ejemplo, algunos datos que resaltan en la tabla 1 son: el tiempo de estabilización del primero mejoró con 3.38s mientras que en el segundo fue de 19.6s. 


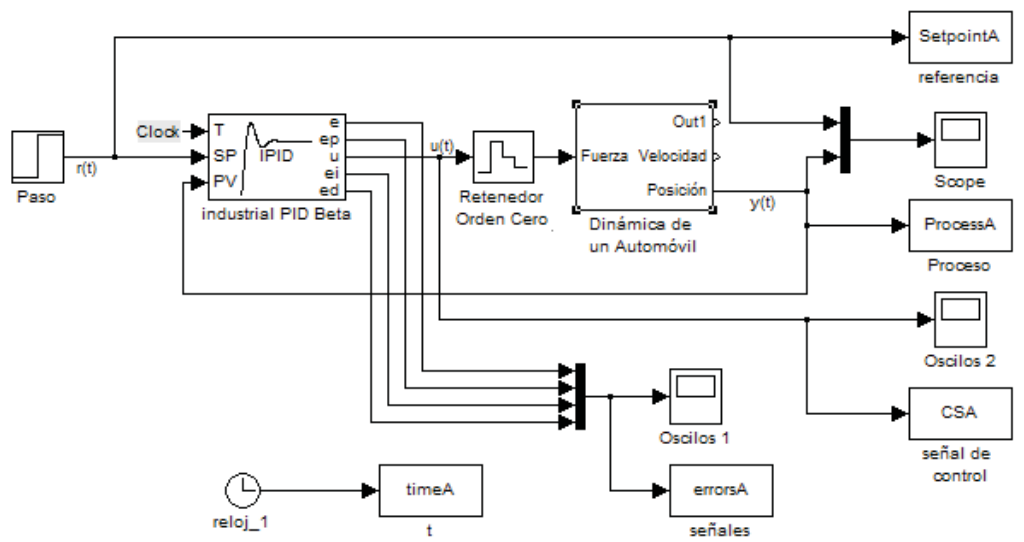

Figura 7. Diagrama de bloques utilizando el bloque «industrial PID Beta» para el sistema de la figura. La señal de entrada es $r(\mathrm{t})$; la de control es $\mathrm{u}(\mathrm{t})$; la de salida es $\mathrm{y}(\mathrm{t})$.

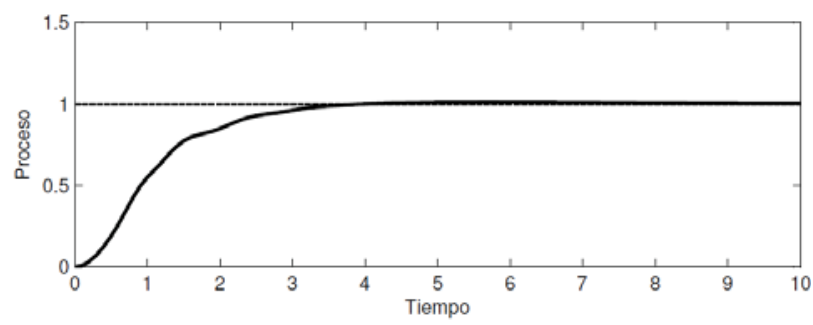

(a)

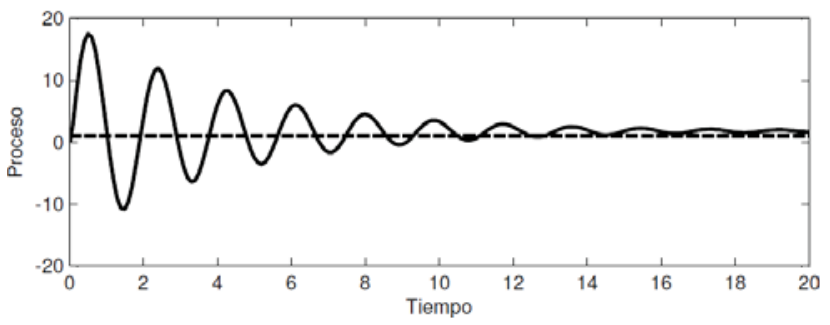

(b)

Figura 8. Respuesta del sistema con controlador PID Práctico (a) y PID Básico (b). La línea punteada: la señal paso; la sólida: el proceso.

Otro ítem interesante es el sobreimpulso que en el PID práctico con 0.98s y en el básico fue de 228.02. Aunque en el primero, el tiempo de elevación fue mayor con 1.94s mientras que en el segundo fue de $0.12 \mathrm{~s}$.

En las figuras 8-10, se pueden observar algunos elementos de cómo modificando la ley básica del PID se logra obtener una mejora en el sistema de control. Por ejemplo, la «patada derivativa» que se observa en la figura $10(b)$, en $t=0$, es minimizada en la figura 10(a).

También, para el mismo caso, el impacto de usar el término derivativo (el cual causa que el sistema no logre estabilizarse rápidamente para el PID básico), es minimizado escogiendo un valor de $\mathrm{N}$ apropiado. La oscilación del término proporcional de la figura 10 (b), con la acción beta, se ve, pues, minimizada en la figura 10 (a). 


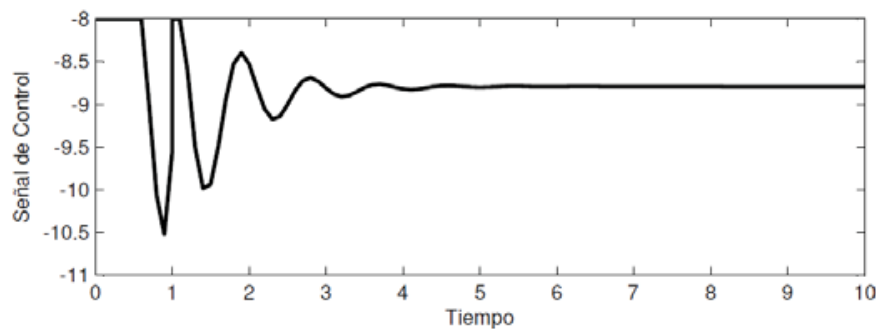

(a)

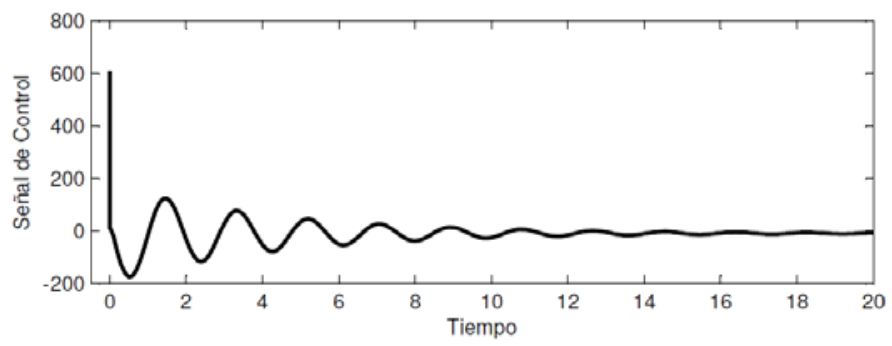

(b)

Figura 9. Respuesta del sistema con controlador PID Práctico (a) y PID Básico (b). Línea sólida: señal de control.

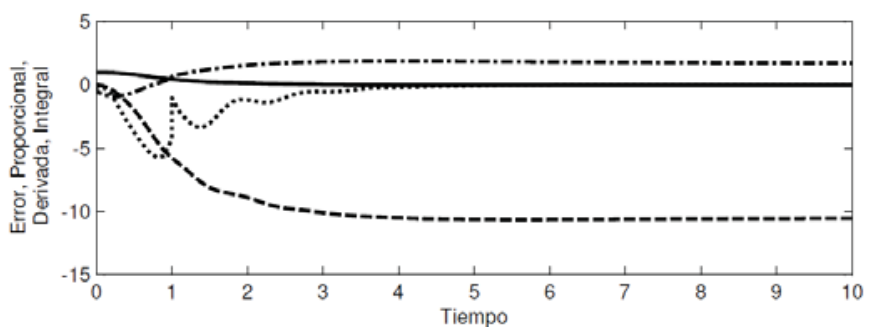

(a)

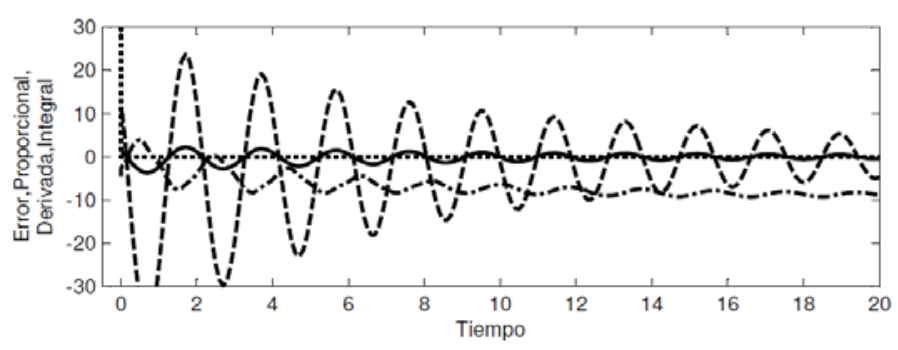

(b)

Figura 10. Desempeño del sistema con PID práctico (a) y básico (b). Línea sólida: señal de error; línea rayaraya: término proporcional; línea punto-punto: término derivativo; línea raya-punto: término integral.

Por último, en el uso del saturador se pueden analizar dos cosas: en la figura 9(a), la amplitud de la señal de control se ve disminuida y eso se refleja en el índice de 
desempeño que, para el PID práctico, fue de 334.40 y el básico 717.11. La segunda es que, cuando esto ocurre, se limita la acción de la integral, es decir, en saturación, la parte integral se anula, lo cual permite que se recupere la acción de control una vez haya alcanzado la referencia. Este comportamiento se observa en la figura 8, el término integral.

\begin{tabular}{|l|c|c|}
\hline Índices de desempeño & PID Avanzado & PID Básico \\
\hline Tiempo de estabilización (s) & 3.38 & 19.6 \\
\hline \hline Tiempo de elevación (s) & 1.94 & 0.12 \\
\hline \hline Sobreimpulso (\%) & 0.84 & 228.02 \\
\hline \hline Energía del error & 0.3593 & 3.3580 \\
\hline \hline Energía de la señal de control & 334.40 & 717.119 \\
\hline \hline IES error cuadrático integral & $9.40 \mathrm{e}-006$ & 0.203 \\
\hline
\end{tabular}

Tabla 1. Resultados de la simulación.

\section{Conclusiones}

- Se analizó la dinámica de un automóvil; mediante él, se explicó el bloque Simulink que viene con la aplicación. Se expusieron las características más importantes: la interfaz de usuario, los algoritmos implementados y su configuración.

- Mediante el ejemplo, se realizaron análisis del comportamiento, a través de la herramienta, de un PID básico y un PID práctico. Esto con el fin de mostrar qué desempeño muestra el sistema al considerar características de implementación de la ley de control de PID.

- Los resultados mostraron una mejora en casi todos los índices de comparación que se permiten analizar con las funciones de la herramienta (como se dijo antes, el bloque Simulink no posee ninguna herramienta de análisis).

- Cabe resaltar que el diseño para el sistema, en general, presenta realmente un pobre desempeño, pues la sintonización de los parámetros $\mathrm{P}$, I y D, en este caso, no fueron los mejores. Sin embargo, pese a esto, al modificar la ecuación básica, se logró un desempeño bastante aceptable (ver tabla 1) lo cual indica, pues, que existen herramientas y estrategias que no solo se basan en la sintonización, sino también en la práctica del control PID.

\section{Referencias}

[1] A. Vissioli, Practical PID Control, 1.a ed., pp. 7-63 Italy: Springer 2006.

[2] D. J. Cooper, University of Connecticut, Practical Process Control. [Online]. Available: www.controlguru.com/

[3] L. Yun, K. Heong, J. C. Gregory, and Y. Chong, «PID Control System Analysis and Design» IEEE Control System Magazine, Feb. 2006, pp. 32-37.

[4] P. G. Iván y G. O. Fabio. Análisis matemático e implementación de un sistema multitanque a escala para docencia e investigación

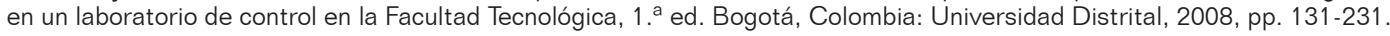


[5] K. J. Astrom and T. Hagglund. Advanced PID Control. Research Triangle Park, ISA-the Instrumentation, System, and Automation Society, 1. ${ }^{\text {a }}$ ed. 2002, pp. 64-92,

[6] J. L. Guzmán, K. J. Astrom, S. Dormido, T. Hagglund, M. Berenguel, and Y. Piguet «Interactive Learning Modules for PID Control». IEEE Trans. Control System Magazine, Oct. 2008, Vol. 8, N. 5, pp 118-134,

[7] Y. Peng, D. Vrancic and R. Hanus. "Anti-Windup, Bumpless and Conditioned Transfer Techniques for PID Controllers». IEEE Trans. Control System Magazine, agosto 1996, Vol. 16, N. 4, pp. 48-56.

[8] C. Bohn and D. P. Atherton, "An Analysis Package Comparing PID Anti-Windup Strategies». IEEE Trans. Control System Magazine, abril 1995, Vol. 15, N. ${ }^{\circ} 2$, pp 34-40.

[9] D. E. Seborg, T. F. Edgar, D. A. Mellichamp. Process Dynamics and Control, United States of America: Wiley 2004, 2. ${ }^{a}$ ed., pp. 324-328.

[10] K. J. Astrom, T. Hagglund. PID controllers: theory, design, and tunning. ISA Press. Research Triangle Park, USA, 1995, pp. 83-86.

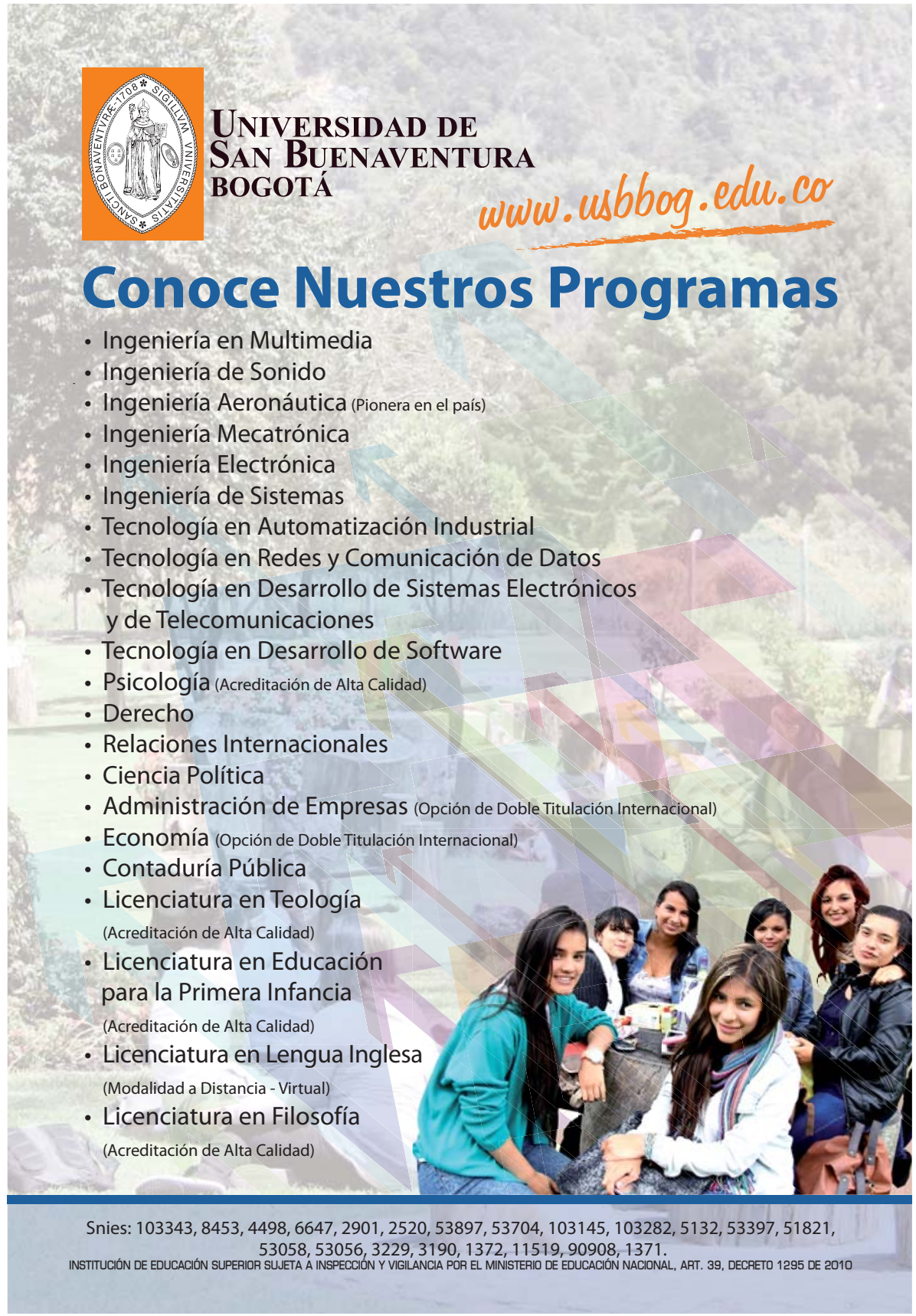

